\title{
Jenseits des Säkularisierungsparadigmas?
}

Eine Auseinandersetzung mit Charles Taylor

\author{
Matthias Koenig
}

Zusammenfassung: Mit seinem Buch A Secular Age hat der Sozialphilosoph Charles Taylor einen aufsehenerregenden Alternativentwurf zum klassischen Säkularisierungsparadigma vorgelegt. Das Ziel dieses Artikels ist es, ihn auf die religionssoziologische Diskussion um Säkularisierung und deren verschiedenen Teilprozesse zu beziehen. Es werden drei Ansprüche formuliert, an denen Alternativentwürfe zu messen sind. Erstens müssen sie verständlich machen, warum ,Säkularität" zu einer so wichtigen Selbstbeschreibungskategorie moderner Gesellschaften werden konnte. Zweitens müssen sie das in Jahrzenten religionssoziologischer Forschung akkumulierte Wissen über Entkirchlichung mit den von Kritikern hervorgehobenen Befunden genuin moderner religiöser Vitalität integrieren. Und drittens müssen sie die vielfältigen Muster der Differenzierung religiöser und politischer Ordnung im Gesellschafts- und Kulturvergleich beschreiben und erklären können. In kritischer Auseinandersetzung mit Taylor werden Grenzen kulturalistischer Theorien moderner Säkularität und bleibende Aufgaben historisch-soziologischer Forschung zu Religion in der Moderne identifiziert.

Schlüsselwörter: Religionssoziologie $\cdot$ Säkularisierung $\cdot$ Sozialtheorie

\section{Beyond the paradigm of secularization?-A discussion with Charles Taylor}

Abstract: Charles Taylor's book A Secular Age is a widely appraised alternative to the classical
paradigm of secularization theory. This article situates this alternative theory within the debate of
sociology of religion on secularization and its sub-components. Three requirements are formulat-
ed that alternative conceptions would have to met. First, they need to understand why ,secularity“
became such a prominent category of self interpretation in modern societies. Second, they have
to be able to integrate years of cumulative sociological research on sub-processes of seculariza-
tion with findings on genuinely modern forms of religious vitality emphasized by the classical

(C) Die Autor(en) 2011. Dieser Artikel ist auf Springerlink.com mit Open Access verfügbar.

Die Fertigstellung dieses Beitrags wurde durch das von der Deutschen Forschungsgemeinschaft finanzierte Lichtenbergkolleg an der Georg-August-Universität Göttingen ermöglicht. Eine frühere Version wurde auf dem Kongress der Deutschen Gesellschaft für Soziologie in Jena (2008) präsentiert. Für wertvolle Hinweise und Anregungen danke ich Christoph Halbig, Hans Joas, InkaLee Huu, Detlef Pollack, Holmer Steinfath sowie den Herausgebern dieser Zeitschrift.

M. Koenig $(\bowtie)$

Institut für Soziologie, Georg-August-Universität Göttingen, Platz der Göttinger Sieben 3,

37073 Göttingen, Deutschland

E-Mail: mkoenig@uni-goettingen.de 
paradigm's critics. And in light of on-going debates over multiple modernities, they would need to describe and explain the varieties of differentiation in societal and cultural comparison. In critical discussion of Charles Taylor's contribution, the limits of culturalist theories of modern secularity as well as some tasks for historical-sociological research on religion in modernity are identified.

Keywords: Sociology of religion $\cdot$ Secularization $\cdot$ Social theory

\section{Einleitung}

Mehr denn je steht die Säkularisierungstheorie in der Kritik. Die Aussage, Modernisierung führe notwendig zu einem Bedeutungsverlust von Religion, war in der Religionssoziologie zwar schon lange gravierenden Einwänden ausgesetzt (vgl. Martin 1965; Glasner 1977; Ausmus 1982; Hadden 1987). Aber erst die jüngere Aufmerksamkeit für fundamentalistische Bewegungen, religiösen Nationalismus und die mit Migration und Globalisierung einhergehende religiöse Pluralisierung hat der Säkularisierungstheorie den einstmaligen Status eines nahezu unbestrittenen Paradigmas entzogen. Zeitdiagnostiker sprechen von einer Renaissance des Religiösen, von „De-Säkularisierung“ oder vom Kommen einer „post-säkularen“ Gesellschaft (Berger 1999; Habermas 2001). Und auch in den Fachdiskussionen von Geschichtswissenschaft, Politologie und Soziologie hat das Säkularisierungsparadigma einen steten Plausibilitätsverlust erlitten (Literaturübersichten bei Borutta 2010; Ebaugh 2002; Gorski und Altınordu 2008; Philpott 2009).

Worauf die Kritik am Säkularisierungsparadigma genau abzielt, ist indessen alles andere als eindeutig. Insbesondere die zeitdiagnostisch motivierte Kritik bleibt zumeist unscharf. Einerseits wirft man der Säkularisierungstheorie vor, sie reproduziere eine zu lineare Meistererzählung der Moderne, welche die vielen Ungleichzeitigkeiten, gegenläufigen Entwicklungen und historischen Brüche der jüngeren Religionsgeschichte übersehe. Andererseits nimmt man genau diese Selbstbeschreibung der Moderne in Anspruch, um für die Gegenwart sodann einen fundamentalen Epochenwandel zu behaupten. ${ }^{1}$ Unscharf bleibt die Kritik aber auch in der fachwissenschaftlichen Diskussion. In der Religionssoziologie umfasste das Säkularisierungsparadigma stets mehrere, im Übergang von traditionalen zu modernen Gesellschaften erfolgende Teilprozesse. José Casanova (1994, S. 20-39), um nur den derzeit einflussreichsten Systematisierungsversuch zu nennen (vgl. aber auch Dobbelaere 1981; Tschannen 1991), fasst unter Säkularisierung die funktionale Differenzierung der Religion von anderen gesellschaftlichen Ordnungen wie Politik, Recht und Wissenschaft, die Privatisierung von Religion sowie den in der SurveyForschung im Vordergrund stehenden Rückgang individueller Religiosität. Die verschiedenen Teilprozesse aber sind von den Einwänden gegen das Säkularisierungsparadigma nicht gleichmäßig betroffen. Sowohl die auf Thomas Luckmann (1991) zurückgehende These des Fortbestehens individualisierter ,unsichtbarer“" Religion als auch die von Rodney Stark und anderen (Stark 1999; Warner 1993) vertretene Markttheorie der Religion,

1 Dass Diagnosen eines ,post-säkularen“ Zeitalters weniger einen Epochenwandel als vielmehr eine Selbstkorrektur intellektueller Wahrnehmungsmuster artikulieren könnten, betont Hans Joas (2004, S. 124); vgl. zur Diskussion auch Philipp (2009) und Torpey (2010). 
deren empirische Belastbarkeit im Übrigen alles andere als gesichert ist (vgl. nur Norris und Inglehart 2004; Pollack 2009), bestreiten lediglich den Rückgang individueller Religiosität, führen gleichzeitig aber Privatisierung und Differenzierung ins Feld, um hier die angebotsabhängige religiöse Vitalität, dort die massenkulturelle Sozialform des Religiösen zu erklären. Casanova selbst hat in Kritik an der Privatisierungsthese die bleibende Bedeutung von Religion in Zivilgesellschaft und öffentlicher Sphäre betont, an der Differenzierungsthese als paradigmatischem Kern der Säkularisierungstheorie aber zunächst festgehalten (Casanova 1994, S. 212). In Reaktion auf Einwände von Talal Asad (1999, S. 179) behauptet er aber nunmehr (Casanova 2008), dass das Auftreten öffentlicher Religion nicht nur Privatisierung, sondern auch Differenzierung in Frage stelle, ja, dass beide Teilprozesse analytisch nur schwerlich zu trennen seien und ohnehin historisch und geographisch multiple Varianten aufwiesen.

Diese Diskussionslage macht deutlich, wie hoch die Anforderungen an eine überzeugende Alternative zum klassischen Säkularisierungsparadigma in der Religionssoziologie inzwischen geworden sind. Mindestens den folgenden drei Ansprüchen müsste sie genügen (vgl. auch Gorski und Altınordu 2008). Erstens müsste sie verständlich machen, warum „Säkularität“ in Diskursen intellektueller Eliten, aber auch in Mentalitäten ganzer Bevölkerungen zu einer so wichtigen und umkämpften Selbstbeschreibungskategorie zumindest der europäischen Moderne werden konnte, woher also die zeitweilige Plausibilität des Säkularisierungsparadigmas herrührte. Zweitens müsste sie die in Jahrzehnten empirischer Forschung akkumulierte Evidenz für den Rückgang individueller Kirchlichkeit in Europa mit den von Kritikern hervorgehobenen Befunden genuin moderner religiöser Vitalität innerhalb und außerhalb Europas in einem kohärenten analytischen Bezugsrahmen integrieren. Dieser müsste es drittens erlauben, die Vielfalt moderner Differenzierungsmuster religiöser und politischer Ordnung, „multiple secularity“ (Katznelson und Jones 2010, S. 20), im Gesellschafts- und Kulturvergleich zu beschreiben und zu erklären.

Wenige Alternativentwürfe erfahren derzeit dieselbe Aufmerksamkeit wie die monumentale Studie A Secular Age des kanadischen Sozialphilosophen Charles Taylor (2007, 2009). ${ }^{2}$ Casanova bezeichnet sie gar als ,the best analytical, phenomenological, and genealogical account that we have of our modern, secular condition" (Casanova in Warner et al. 2010, S. 265). Seine enorme Resonanz verdankt Taylors Alternativentwurf nicht zuletzt dem Umstand, dass er das säkulare Selbstverständnis der Moderne ins Zentrum der Debatte rückt. Säkularisierung bezeichne nicht nur die Differenzierung der Religion von anderen gesellschaftlichen Ordnungen und die damit verbundene Privatisierung (,Säkularität 1“) oder den Rückgang individuellen Glaubens (,Säkularität 2“), sondern vor allem auch die Konstruktion eines „framework of understanding“ (S. 3, 34), eines kulturellen Horizonts, der nicht-religiöse und religiöse Praktiken gleichermaßen bestimmt (,Säkularität 3“). Das klassische Säkularisierungsparadigma versucht Taylor mithin zu überbieten,

2 Alle Seitenangaben beziehen sich im Folgenden, wenn nicht anders angegeben, auf die englischsprachige Originalausgabe von A Secular Age. Aus der umfangreichen Rezensionsliteratur seien hier die sozialwissenschaftlichen Kommentare von Calhoun (2008), Hurd (2008), Joas (2009) und McLennan (2008) hervorgehoben. Vgl. ferner den Blog des Social Science Research Council (blogs.ssrc.org/tif) sowie die Beiträge in Warner et al. (2010). 
indem er es als Bestandteil eines historisch zu rekonstruierenden „immanten Rahmens“ darstellt, innerhalb dessen die anderen Teilprozesse von Säkularisierung überhaupt erst möglich werden.

Ein solch ambitionierter Alternativentwurf verdient auch in der Religionssoziologie ausführlich diskutiert zu werden. Angesichts von Taylors hochselektiver Literaturrezeption erfordert dies zunächst, seine Säkularitätstheorie interpretatorisch in die religionssoziologische Diskussionslage einzuordnen, um sodann kritisch zu fragen, inwieweit sie den oben formulierten Ansprüchen genügen kann. Genau dies ist das Anliegen des folgenden Artikels. In einem ersten Schritt werden unter Rückgriff auf die Werkgeschichte die grundbegrifflichen Weichenstellungen nachvollzogen, die es Taylor erlauben, eine reflexive Beobachterperspektive auf den immanenten Rahmen einzunehmen, und die ihn zu einer im Kern kulturalistischen Theorie moderner Säkularität führen, die, trotz offenkundiger Parallelen zur phänomenologischen Tradition des Faches, zur Soziologie explizit auf Distanz geht (Abschn. 2). In einem zweiten Schritt ist Taylors Säkularitätstheorie daraufhin zu befragen, inwieweit sie die vermeintlich widersprüchlichen Befunde von Entkirchlichung einerseits, religiöser Vitalität andererseits zu integrieren vermag (Abschn. 3). Und schließlich ist zu diskutieren, welchen Ertrag seine Rekonstruktion des immanenten Rahmens für die gesellschafts- und kulturvergleichende Analyse von Mustern der Differenzierung religiöser und politischer Ordnung abwirft (Abschn. 4). Eine solch ausführliche Auseinandersetzung mit Taylors Alternativentwurf zum konventionellen Säkularisierungsparadigma lohnt nicht nur deshalb, weil seine Rekonstruktion des immanenten Rahmens die religionssoziologische Säkularisierungsdebatte um eine weitere Facette bereichert (,Säkularität 3“), sondern auch, weil die Defizite dieses Unterfangens auf bleibende Aufgaben religionssoziologischer Forschung hinweisen.

\section{Säkularität als kulturelle Konstruktion - grundbegriffliche Weichenstellungen}

Eine Alternative zum klassischem Säkularisierungsparadigma, dies wurde oben als erster Anspruch formuliert, muss erklären können, warum „Säkularität“ zu einer so wichtigen und umkämpften Selbstbeschreibungskategorie moderner Gesellschaften werden konnte. Haben begriffsgeschichtliche und diskursgenealogische Studien dazu bereits wichtige Vorarbeiten geleistet (z. B. Asad 1993; 2003; Milbank 1990), so versucht Taylor den kulturellen Horizont nicht nur der Intellektuellendiskurse, sondern auch der Mentalitäten ganzer Bevölkerungen auszuleuchten. Dies Anliegen verfolgt Taylor, indem er, der phänomenologischen Tradition der Soziologie nicht unähnlich, sparsame formal-anthropologische Voraussetzungen einführt, aus denen sich eine radikale Historisierung der für die Moderne charakteristischen Konzeptionen natürlicher, sozialer und subjektiver Welt ergibt. Vor allem soziologische Säkularisierungstheorien kritisiert Taylor dafür, dass sie den immanenten Rahmen in Kategorien wie „Entzauberung“ oder „Rationalisierung“ naturalisiert und mithin die Kontingenz seiner kulturellen Konstruktion verdeckt hätten.

\subsection{Formale Anthropologie und Kulturtheorie der Moderne}

Seit seinem frühen Hegel-Buch (Taylor 1975) hat sich Taylor darum bemüht, eine philosophische Anthropologie zu formulieren, die der Einsicht Rechnung trägt, dass Menschen 
sinnorientierte Wesen sind und sich selbst daher in den Kategorien eines szientifischen Naturalismus nicht angemessen verstehen können (vgl. insbesondere Taylor 1992). Taylors Denkbewegung kreist, wie in der Sekundärliteratur vielfach hervorgehoben wurde, um zwei Achsen: eine formale Anthropologie und eine hermeneutische Rekonstruktion der Kultur der Moderne (vgl. Rosa 1998, S. 24 f.; Smith 2002, S. 7). Sie erinnert darin an Thomas Luckmanns Verschränkung von Konstitutions- und Konstruktionsanalyse sozialer Wirklichkeit, worin erstere in der phänomenologischen Rekonstruktion invarianter Strukturen subjektiver Sinnorientierung, letztere in der im eigentlichen Sinne soziologischen Analyse historisch variabler sozialer Wirklichkeiten besteht (z. B. Luckmann 2002, S. 51). Tatsächlich stützt sich auch Taylor wie Luckmann in seiner formalen Anthropologie auf das phänomenologische Erbe (Merleau-Ponty, Husserl, Heidegger). Zwar ist er für die Paradoxien und Probleme von Husserls Methode der reinen Reduktion sensibel, bemüht sich aber gleichwohl um eine minimale Definition dessen, was Menschsein ausmacht. Dazu gehöre die körperliche Verfasstheit menschlicher Erfahrung als praktischem Umgang mit Dingen, die sprachliche Verfasstheit menschlicher Expressivität und die moralische Orientierung menschlichen Handelns an „starken Wertungen“, worunter er die in einer kulturellen Lebensform eingebetteten Wertungen versteht, die im Unterschied zu unseren bloßen Wünschen dasjenige beinhalten, was uns als wünschenswert erscheint, und die damit für unsere personale Identität konstitutiv sind. ${ }^{3}$ Diese formal-anthropologischen Annahmen sind für Taylor deswegen wichtig, weil sie die historisch-narrative Rekonstruktion moderner Kultur, wie er sie vor allem in den Quellen des Selbst (Taylor 1995) unternommen hat, auf das Gleis einer kulturalistischen Theorie der Moderne setzen (vgl. dazu Reckwitz 2001, S. 478-522).

Die Verschränkung von formaler Anthropologie und Kulturtheorie der Moderne kennzeichnet auch A Secular Age, in dem Taylor sein tiefes Interesse am Schicksal religiöser Traditionen in der Moderne (vgl. Taylor 1999, 2002; dazu Kühnlein 2008) zum Leitthema macht. Auch hier stützt er sich auf eine formalanthropologische Prämisse, die Prämisse nämlich, wonach Menschen ihr Leben stets bezogen auf Vorstellungen von Erfüllung („fullness“) erfahren: „Somewhere, in some activity, or condition, lies a fullness, a richness“ (S. 5) oder, wie es an anderer Stelle heißt, ,for any liveable understanding of human life, there must be some way in which this life looks good, whole, proper, really being lived as it should“" (S. 600, Hervorhebung vom Verf.). Diese Vorstellungen wirkten nicht nur in den außeralltäglichen Erfahrungen von Erfüllung, sondern auch in den negativen Erfahrungen von Verlust oder Scheitern und selbst in den Erfahrungen des alltäglichen und doch zugleich gerichteten Lebens. Dass Menschen auf irgendeine Art und Weise an Erfüllung orientiert leben, ist für Taylor invarianter Bestandteil der conditio humana. Wie Erfüllung erfahren wird, ist hingegen historisch äußerst variabel. Idealtypisch unterscheidet Taylor religiösen Glauben (,,belief") und Unglaube („unbelief“) als zwei Varianten gelebter Erfahrung von Erfüllung (S. 8). In der Variante des Glaubens werde die Fülle des

3 Vgl. dazu Joas (1997, S. 200) sowie Rosa (1998, S. 109). Man könnte Taylors Konzept „starker Wertungen“" auch auf den Begriff letzter Werte (,ultimate values“) des frühen Parsons (1935) beziehen. Allerdings setzt Taylor insoweit andere Akzente als jene Wertungen nicht einfach in einem kulturellen System vorgefunden werden, sondern aus dessen Wechselspiel mit den praktischen Erfahrungen der Handelnden hervorgehen; vgl. dazu Joas (1997, S. 32 und 212). 
Lebens als empfangen erfahren, die letzten Ziele (,final goals“) des Lebens würden mithin als transzendent verortet, etwa in der Liebe Gottes. Eine rein immanente Bestimmung erhielten sie demgegenüber in der Variante des Unglaubens, die Taylor im ,selbstgenügsamen“ oder „exklusiven Humanismus” realisiert sieht - ,a humanism accepting no final goals beyond human flourishing, nor any allegiance to anything else beyond this flourishing” (S. 18 f.). Die Fülle des Lebens werde hier in menschlichem Gedeihen (,human flourishing") selbst gesucht und nicht als empfangen, sondern, sei es qua Vernunft (Kant), qua Gefühl (Romantik) oder qua Heroismus (Nietzsche), als erreichbar erfahren.

Die Unterscheidung von Glauben und Unglauben als zwei Varianten der formalanthropologisch invarianten Offenheit des Menschen für Erfahrungen von Erfüllung bindet Taylor ganz unmissverständlich an die Unterscheidung von Transzendenz und Immanenz. Diese Unterscheidung ist in der religionstheoretischen Debatte geläufig (vgl. nur Pollack 1995), erfährt bei Taylor aber einen spezifischen Zuschnitt. Erstens hält Taylor aufgrund seiner Kritik am szientifischen Naturalismus dezidiert an einer anthropologisch fundierten Religionstheorie fest; die von Luhmann (2000, S. 13, 77) geforderte Wende zur einer Kommunikationstheorie der Religion und ihres Transzendenz/Immanenz-Codes könnte Taylor daher nicht mitvollziehen. Zweitens verwendet Taylor einen starken Transzendenzbegriff. Während etwa Luckmann (1991) Erfahrungen von Transzendenz und damit auch Religiosität schon konstitutionsanalytisch invariant setzt, sind für Taylor lediglich die Erfahrungen der Erfüllung formalanthropologisch vorgegeben. Religiöser Glaube als eine ihrer möglichen Varianten bestehe nicht im bloßen Überschreiten des Alltags auf menschliche letzte Ziele hin, sondern im Transzendieren jener menschlichen Ziele selbst. Dabei legt Taylor den Akzent nicht nur auf das kognitive Für-Wahr-Halten einer transzendenten Realität (z. B. Gottes), sondern auch auf die normative Orientierung an Vorstellungen eines erfüllten Lebens, die Güter jenseits des menschlichen Gedeihens (z. B. die Liebe Gottes) beinhalten und dadurch eine Transformation des Menschen über dessen eigene Perfektion hinaus ermöglichen (S. 20, 510).

\subsection{Der immanente Rahmen und die Grenzen soziologischer Säkularisierungstheorie}

Die soweit eingeführten grundbegrifflichen Weichenstellungen helfen bei der Klärung dessen, was Taylor unter ,Säkularität 3“ versteht, nämlich eine Situation, in der die Suspension aller Güter jenseits menschlichen Gedeihens vorstellbar, in der, anders gesagt, Unglaube zu einer echten Option geworden ist. Das säkulare Zeitalter unterscheide sich von einem Zeitalter, in dem religiöser Glaube der selbstverständliche Modus für Erfahrungen der Erfüllung war. Jenes Zeitalter des Glaubens, wie es die mittelalterliche Christenheit prägte, setzte spezifische Konzeptionen der natürlichen, der subjektiven und der sozialen Welt voraus. Die natürliche Welt wurde als Kosmos und Schöpfung Gottes gesehen, das Selbst stellte man sich als offen für das Wirken transzendenter Mächte vor (,porous self"), und die soziale Welt war durch hierarchische Beziehungen strukturiert, die in eine höhere Ordnung (die Kirche) und Zeit (die Heilsgeschichte) eingebettet waren. Taylors These ist, dass das säkulare Zeitalter demgegenüber aus einer Entzauberung (,disenchantment“) aller drei Weltkonzeptionen hervorgeht. ${ }^{4}$ An die Stelle des Kosmos

4 Dass Taylor hier von „Entzauberung“ spricht, läuft seinen eigenen Absichten, wie im Folgenden deutlich werden sollte, zuwider; vgl. Milbank in Warner et al. (2010, S. 57) und zur komplexen Begriffsgeschichte von „Entzauberung“ Lehmann (2009). 
tritt die Vorstellung eines mechanistischen Universums, es entstehen Bilder eines abgeschlossenes Selbst („,buffered self“) und die soziale Welt wird als eine Gesellschaft imaginiert, zu der alle einen direkten und gleichen Zugang besitzen, in der Identität nicht mehr relational, sondern kategorial (z. B. als nationale Zugehörigkeit) gedacht ist und deren Geschichte sich in einer zukunftsoffenen homogenen Zeit vollzieht. Durch die Entzauberung der drei Weltkonzeptionen werde, so Taylor, ein immanenter Rahmen (,immanent frame") aufgespannt, der neue Erfahrungen von Erfüllung ermögliche, nämlich genau diejenigen des Unglaubens, und der gleichzeitig die einstige Naivität des Glaubens verunmögliche. Das Signum des säkularen Zeitalters sei daher, dass auch religiöser Glaube stets als Option erfahren werde (S. 14).

Bis hierher scheint es, als reformuliere Taylor eine recht konventionelle Säkularisierungstheorie, wie sie insbesondere aus den älteren soziologischen Schriften von Berger (1973, 1992) bekannt ist. Tatsächlich weist Taylors Vorgehensweise einige Parallelen zu Bergers Variante der phänomenologisch fundierten Wissenssoziologie auf. Seine theoretische Unterscheidung der drei Bedeutungsvarianten von Säkularisierung entspricht Bergers dreigliedriger Konzeption von Säkularisierung als eines Vorgangs auf der Ebene des individuellen Bewussteins, der gesellschaftlichen Institutionen und der kulturellen Weltbilder (Berger 1973, S. 103 f.). Und vor allem wenn Taylor den optionalen Charakter religiösen Glaubens in der Moderne betont, fühlt man sich unmittelbar an Bergers Zwang zur Häresie (1992) erinnert, wonach der Zerfall eines die Gesellschaft religiös überwölbenden heiligen Baldachins und die damit einhergehende Weltbild-Pluralisierung religiösen Glauben als kontingente Wahlmöglichkeit erfahren lassen.

Dass Taylor in seiner Analyse des säkularen Zeitalters eigene Wege geht, wird indessen deutlich, sobald man die genetische Frage nach dessen Entstehung aufwirft. Konventionelle Säkularisierungstheorien nehmen, so Taylor, die Form einer Subtraktionserzählung (,subtraction story“, S. 26) an. Danach zerreißt die neuzeitliche Wissenschaft mit ihrem Drang zur Rationalisierung die Vorhänge der Unvernunft, lüftet die Geheimnisse des Zaubergartens, erkennt das Universum, Selbst und Gesellschaft in ihrer eigentlichen Natur und entzieht dem religiösen Glauben damit seine einstige Plausibilität. ${ }^{5}$ Im Zentrum von Taylors Kritik steht die neo-orthodoxe Säkularisierungstheorie der Soziologie (Bruce 1996, 2002; Wallis und Bruce 1992). Ihr wirft er vor, sie nehme mit ihrem impliziten Stadienbewusstsein und dem Gestus einer neutralen Beobachterposition unreflektiert eine Epistemologie in Anspruch, die doch selbst ein Ergebnis kultureller Konstruktionsprozesse sei (S. 428, 436). Taylors Kritik an den Subtraktionserzählungen soziologischer Säkularisierungstheorie zielt bei näherem Hinsehen auf ihr mangelndes Kontingenzbewusstsein und ihre reduktionistische Religionstheorie.

Mangelndes Kontingenzbewusstsein kennzeichne die konventionelle Säkularisierungstheorie insofern, als sie den immanenten Rahmen naturalisiere, den kontingenten Vorgang seiner historischen Konstruktion jedoch ausblende (S. 556-566). „The subtraction story gives too little place to the cultural changes wrought by Western modernity, the way in which it has developed new understandings of the self, its place in society, in space

5 Man mag hier auch an die Aneignung von Webers Entzauberungsbegriff in Habermas' (1981) Theorie kommunikativer Rationalisierung denken, mit der sich Taylor wiederholt und kritisch auseinandergesetzt hat. 
and in time. It fails to see how innovative we have been; its tendency is to see modernity as the liberating of a continuing core of belief and desire from an overlay of metaphysical/ religious illusion which distorted and inhibited it" (S. 573, Hervorhebung vom Verf.). Das säkulare Zeitalter in seiner Genese zu verstehen, erfordert aus Taylors Sicht mithin, die Konstruktion des immanenten Rahmens als eines geteilten kulturellen Horizonts, eines neuen heiligen Baldachins, wenn man so will, historisch nachzuvollziehen.

Die konventionelle Säkularisierungstheorie leidet Taylor zufolge aber auch unter einer reduktionistischen Religionstheorie. Unstrittig ist für Taylor zunächst, dass religiöse Erfahrungen von Erfüllung, die Transzendenz voraussetzen und auf eine Transformation des Menschen zielen, unter dem Eindruck des immanenten Rahmens durchaus an Plausibilität eingebüßt hätten (,Säkularität 2“). Strittig sei vielmehr die Erklärung dieses Bedeutungsverlusts und, komplementär dazu, die Einschätzung der möglichen Zukunft von Religion. Weder sieht Taylor den Prozess gesellschaftsstruktureller Modernisierung als hinreichende Bedingung für den Bedeutungsverlust religiösen Glaubens, noch sieht er in der Indifferenz gegenüber Erfahrungen der Erfüllung den notwendigen Fluchtpunkt jenes Prozesses. Beide Behauptungen unterstellten der Religion nämlich fälschlicherweise einen epiphenomenalen Charakter: „I don’t accept what often seems to be an unspoken premise about human motivation which underlies this master narrative of secularization. In particular, I hold that religious longing, the longing for and response to a more-than-immanent transformation perspective (...) remains a strong independent source of motivation in modernity" (S. 530, Hervorhebung vom Verf.). Taylor vertritt also religionsphilosophisch gesprochen eine anti-reduktionistische Position, wonach religiöse Überzeugungen sich nicht auf psychische oder soziale Faktoren reduzieren lassen, sondern eigene motivationale Kraft entfalten können. ${ }^{6}$ Genau diese Position aber ermöglicht es, auch unter Bedingungen des immanenten Rahmens Momente religiöser Vitalität zu identifizieren.

Beide Vorwürfe an die Soziologie, ihr mangelndes Kontingenzbewusstein und ihre reduktionistische Religionstheorie, sind sicherlich etwas überzogen und pauschal geraten. Taylors grundbegriffliche Weichenstellungen ähneln nicht nur denjenigen der phänomenologischen Soziologie, sie zielen auch in ähnliche Richtungen wie das ,strong program“ der neueren Kultursoziologie (Alexander 2003; Reckwitz 2001) und lassen sich umstandslos in die auf Weber zurückgehende Tradition der verstehenden Soziologie übersetzen. Auch dort ist man bekanntlich für die motivationale Kraft religiöser Ideen offen (Schluchter 1988). Und auch dort kann man die Moderne als ein kulturelles Programm konzipieren, dessen innerweltlicher Zuschnitt die Vorzeichen religiösen Glaubens grundlegend verändert hat (vgl. Eisenstadt 1998; Meyer et al.1987). Dennoch lässt sich Taylors Soziologiekritik nicht leicht beiseite schieben. Sie impliziert nämlich letztlich, dass die Einnahme einer (vermeintlich) neutralen Beobachterperspektive das Nachdenken über den immanenten Rahmen von vornherein verengt und daher aufgegeben werden muss. Das Nachdenken über den immanenten Rahmen zu weiten, und genau das ist die pragmatische Absicht von Taylors gesamtem Unternehmen, verlangte vielmehr, die

6 Gelegentlich favorisiert Taylor gar die Position eines theistischen Realismus, wonach religiöser Glaube eine dem exklusiven Humanismus überlegene Antwort auf eine transzendente Realität sei (S. 437 und 768); dazu Smith (2010, S. 113). 
Vielfalt der in der Moderne entstandenen religiösen und nicht-religiösen Teilnehmerperspektiven gleichermaßen hermeneutisch zu erschließen. ${ }^{7} \mathrm{Ob}$ die Soziologie gut beraten wäre, Taylors hermeneutischem Vorgehen zu folgen, oder ob sie an ihrer disziplinären Beobachterperspektive festhalten sollte, muss hier zunächst offenbleiben. Taylors Alternativentwurf zum konventionellen Säkularisierungsparadigma bietet jedenfalls Anlass genug, diesbezüglich die eigenen impliziten Hintergrundannahmen offenzulegen und zu prüfen. Gerade formalanthropologische Fragen werden mit Blick auf den Diskussionsstand in den Naturwissenschaften, aber vor allem in der Philosophie zu klären sein (vgl. Smith 2010).

Dem ersten der eingangs genannten Ansprüche wird Taylor, das sollte deutlich geworden sein, weitgehend gerecht. Das säkulare Selbstverständnis der (westlichen) Moderne in seinem historischen Gewordensein zu erschließen, ist geradezu der Kern seines Alternativentwurfs zur konventionellen Säkularisierungstheorie. Zu fragen bleibt indessen, inwieweit Taylors hermeneutische Rekonstruktion des immanenten Rahmens auch den beiden anderen Ansprüchen gerecht wird.

\section{Der immante Rahmen und die Religionsgeschichte der Moderne}

Ein Alternativentwurf zum konventionellen Säkularisierungsparadigma, so der zweite eingangs formulierte Anspruch, muss die empirischen Befunde des Bedeutungsrückgangs individuellen Glaubens (,Säkularität 1") mit konkurrierenden Befunden religiöser Vitalität integrieren können. Um es gleich vorwegzunehmen: A Secular Age bietet durchaus etliche Einsichten, warum der immanente Rahmen nicht nur Plausibilitätsverluste religiösen Glaubens nach sich ziehen, sondern auch religionsproduktiv wirken konnte.

Diese Einsichten gewinnt Taylor seinen hermeneutischen Intentionen entsprechend nicht auf dem Wege deduktiver Theoriebildung, sondern auf dem Wege einer Erzählung über die Entstehung gegenwärtiger Erfahrungsformen. Eine solche Erzählung kann sich nicht auf eine konventionelle Ideen- oder Geistesgeschichte beschränken, sondern muss den kulturellen Horizont moderner Gesellschaften insgesamt ausleuchten. Maßgeblich dafür ist der bereits an anderer Stelle (Taylor 2004) eingeführte Begriff sozialer Imaginationen (,social imaginaries“). Mit sozialen Imaginationen, z. B. den Ideen von Marktökonomie, öffentlicher Sphäre oder demokratischer Selbstregierung, meint Taylor implizite Ordnungsvorstellungen, die in dreifacher Weise über intellektuell artikulierte Theorien hinausgehen: Sie bestehen (a) aus mehrfach übereinander geschichteten Hintergrundannahmen faktischer und normativer Art, die (b) von ganzen Gesellschaften geteilt werden und (c) gemeinsame Praktiken ermöglichen (S. 171; vgl. auch Taylor 2004, S. 23). Will man den Wandel sozialer Imaginationen erklären, aus dem der kulturelle Horizont der

$7 \mathrm{Zu}$ ihnen gehört ausdrücklich die Teilnehmerperspektive des (katholisch) Glaubenden (,perspective as a believer“, S. 437), der Taylor sich persönlich verpflichtet fühlt; vgl. dazu bereits das Vorwort zur englischen Übersetzung von Marcel Gauchets Désenchantement du monde (Taylor 1997) sowie den Beitrag „A Catholic Modernity“ (Taylor 1999). Taylors normative Absicht ist dabei, durch Rückbesinnung auf den christlichen Glauben das Autonomiestreben der Moderne neu zur Geltung zu bringen; vgl. Joas (2004, S. 100) und Smith (2002, S. 236). 
Moderne entstanden ist, muss man nachzeichnen, wie zunächst neue Theorien entstehen, sich mit begrenzten Praktiken von Eliten verbinden, sich sodann auf verschiedenste Handlungsfelder ausbreiten und schließlich zu selbstverständlich vorausgesetzten Hintergrundannahmen ganzer Bevölkerungen werden. Taylors Säkularitätsnarrativ operiert damit auf zwei, in seiner Darstellung allerdings nicht immer leicht zu trennenden Ebenen. Auf der Ebene artikulierter Theorien geht es zunächst um die Entstehung des exklusiven Humanismus, durch den der „Unglaube“ zumindest bei intellektuellen Eliten zu einer möglichen Option wurde. Auf der Ebene sozialer Imaginationen wird sodann nachvollzogen, wie jene Theorien soziale Praktiken zu deuten halfen und schließlich Eingang in die kulturellen Hintergrundannahmen moderner Gesellschaften fanden. Auf beiden Ebenen werden im Folgenden die wichtigsten Stationen auf dem langen Weg vom christlichen Mittelalter zum säkularen Zeitalter genannt, um zu prüfen, inwieweit Taylors Erzählung die Widersprüche der modernen Religionsgeschichte einzufangen gestattet.

\subsection{Reform, exklusiver Humanismus und die Vervielfältigung der Optionen}

Zunächst ist zu betonen, wo Taylors Erzählung ihren Ausgang nicht nimmt. Ausdrücklich verwirft er Erklärungen von exklusivem Humanismus und moderner Säkularität, die auf eine Rückwendung zur Antike abstellen. Platonische, stoische und aristotelische Traditionen widerstünden einer radikalen Entzauberung, und antike Vorläufer des Naturalismus, wie etwa Epikur oder Lukrez, entbehrten jeglicher Antriebe zu aktiver Weltgestaltung, die doch ein zentrales Merkmal des immanenten Rahmens seien (S. 27). Den Ausgangspunkt seiner Säkularisierungserzählung verortet Taylor vielmehr im Christentum, ähnlich wie schon in Quellen des Selbst und (auch wenn dies nicht immer offengelegt wird) auf der Linie der Weberianischen Tradition der Religionssoziologie (vgl. nur Schluchter 1979; Gauchet 1985; Meyer et al. 1987; Eisenstadt 2003). Sein besonderes Interesse gilt dem Drang zur Reform, der im Hoch- und Spätmittelalter einsetzte und in der Epoche von protestantischer Reformation und katholischer Gegenformation seinen Höhepunkt erreichte. Gestützt auf einschlägige mediävistische Literatur (Jean Delumeau, Keith Thomas etc.) hebt Taylor die Ordnungswut (,,rage for order", S. 63) hervor, die sich im Klerus, den Ordensgemeinschaften und heterodoxen Bewegungen Bahn brach und den das Mittelalter bis dato prägenden Gegensatz einer anspruchsvollen religiösen Virtuosenethik und einer von magischen Praktiken und Momenten karnevalesker „Anti-Struktur“ (Turner) durchsetzten Laienreligiosität zu überwinden versuchte; und zwar durch Steigerung der Standards von Rechtgläubigkeit. Aktivismus, Uniformismus, homogene Organisation und Rationalismus sind die wichtigsten Elemente dieses Drangs zur Reform, der nicht nur den kulturellen Vorgang der „Entzauberung“ vorantrieb, sondern auch, vor allem im calvinistischen Zweig der Reformation, neue Formen religiöser und gesellschaftlicher Disziplinierung schuf (S. 77). ${ }^{8}$

Von diesem Ausgangspunkt zeichnet Taylor zunächst die Entstehung des exklusiven Humanismus als elaborierter Theorie moralischer Ordnung nach. Der christliche Drang

8 Angesichts der Bedeutung, die Taylor der Verrechtlichung von Sozialbeziehungen in der Moderne zumisst, überrascht es, dass er die rechtsgeschichtlichen Arbeiten von Harold Berman (1983, 2004) zur Gregorianischen Reform und deren Folgen nicht erörtert. 
zur Reform habe neue, gebildete und teils aus der kirchlichen Hierarchie freigesetzte intellektuelle und politische Eliten entstehen lassen, die ab dem 16./17. Jahrhundert die Bühne betraten und neue intellektuelle Dialekte in Wissenschaft, Ethik und Kunst schufen, Dialekte, die, wie Taylor gegenüber konventionellen Subtraktionserzählungen hervorzuheben nicht müde wird, zunächst noch durchaus theologisch verankert waren. So entspringe das Interesse an Natur in den entstehenden Wissenschaften nicht etwa einer Abkehr, sondern umgekehrt einer Hinwendung zu Gott. Und im neo-stoischen Naturrechtsdenken (Grotius, Locke) werde eine erste Version der modernen Theorie moralischer Ordnung formuliert, die mit ihren Prinzipien von Rechten, Freiheit, Gleichheit und gegenseitigem Nutzen (S. 170) einerseits den exklusiven Humanismus vorbereitete, andererseits aber die prinzipiengeleitete Gestaltung der Gesellschaft noch als Auftrag Gottes an die menschliche Vernunft deuten konnte (S. 126-127).

Den radikalen Durchbruch zum exklusiven Humanismus sieht Taylor im Deismus an der Wende vom 17. zum 18. Jahrhundert, als sich im philosophischen und teils auch theologischen Diskurs eine weitreichende anthropozentrische Wende vollzog (S. 222). Vorstellungen eines über Vorsehung, Gnade oder Wunder vermittelten Wirkens Gottes würden zur Konzeption eines distanzierten Schöpfergottes ausgedünnt, der Mensch werde zum unabhängigen und freien Subjekt aktiver Weltgestaltung und Güter jenseits menschlichen Gedeihens gerieten gänzlich aus dem Blick. Gestützt würde diese anthropozentrische Wende durch Vorstellungen von einer unpersönlichen Ordnung in der Natur und vor allem in der Gesellschaft, die nicht mehr beziehungs-, sondern regelförmig imaginiert werde (S. 271, 282). Ideen einer Natur- oder Vernunftreligion rundeten das Set deistischer Annahmen ab (S. 292). Von ihnen ausgehend sei es nur noch ein kleiner Schritt zum exklusiven Humanismus, in dem Güter jenseits menschlichen Gedeihens gänzlich aus dem Blick geraten (S. 245) und, zumal angesichts der zunehmenden Kritik kirchlicher Religion, Unglauben zu einer plausiblen Option werde. Dass jener Humanismus seine Herkunft aus binnenchristlichen Transformationen nicht verleugnen kann, zeigt sich nach Taylor insbesondere an der Idee universeller gegenseitiger Wohltätigkeit (,,benevolence"); einer um ihre transzendenten Bezüge beschnittenen (und insofern korrumpierten) Version christlicher Agapè. Diese Herkunft zu negieren, und das kennzeichnet das Selbstverständnis des exklusiven Humanismus, erfordert daher ein „Stadienbewusstsein“, das die Gegenwart als Fortschritt und jede Rückbesinnung auf Transzendenz als Rückschritt deutet und durch jene Subtraktionserzählungen der Säkularisierung plausibilisiert wird, die in der klassischen Soziologie kanonisiert wurden (S. 289).

Die Entstehung des exklusiven Humanismus als Alternative zum Christentum sieht Taylor indessen nur als Auftakt zum säkularen Zeitalter. Wichtig für die Stabilisierung des immanenten Rahmens, in dem Glaube und Unglaube reflexiv als Optionen erfahren würden, sei nämlich die stetige Diversifikation möglicher Welthaltungen. Nicht nur die aus dem Drang zur Reform hervorgehende christliche Orthodoxie, sondern auch der exklusive Humanismus hätten verschiedenste Kritiken und Gegenkritiken erzeugt. Diese zeichnet Taylor entlang mehrerer ethischer Kontroversen in Philosophie, Literatur und Kunst von der Romantik bis ins späte 19. Jahrhundert detailreich nach. Die Fülle an Positionen, die dabei entsteht, oszilliert letztlich um drei Pole: die religiöse Option des Glaubens, sei es in orthodoxen oder liberalen Varianten, den schon genannten exklusiven Humanismus (Utilitarismus, Kant) und den von Nietzsche ins Spiel gebrachten Herois- 
mus (S. 636). Auf Taylors hellsichtige Diskussion der Dilemmata, an die alle diese drei Optionen in ihren Sichtweisen von menschlichem Gedeihen, Leiden, Gewalt und Tod gleichermaßen stoßen, muss hier nicht eingegangen werden. Festzuhalten ist lediglich, dass die Diversifizierung möglicher Welthaltungen deren optionalen Charakter schärfer hervortreten lässt.

\subsection{Soziale Imaginationen und moderne Religionsformen}

Damit ist bereits die zweite Ebene der Säkularitätserzählung betreten, auf der weniger die artikulierten Theorien moralischer Ordnung, sondern breitenwirksame soziale Imaginationen im Blick sind. Zunächst erläutert Taylor, wie sich im Gefolge von konfessioneller Disziplinierung und frühneuzeitlicher Staatsbildung unter den Eliten neue Praktiken der Zivilität entwickelt hätten, die einen günstigen Resonanzboden für die oben angesprochene anthropozentrische Wende dargestellt hätten und den Beginn eines dreistufigen Übergangs von Theorien moralischer Ordnung zu sozialen Imaginationen markierten (S. 237).

Die erste Stufe bezeichnet Taylor als Ancien Régime (S. 438). Damit ist ein Zustand gemeint, in dem die von Eliten formulierten neuen Ordnungstheorien noch mit vormodernen sozialen Imaginationen koexistieren. Das Ancien Régime kann bereits als Folge des christlichen Drangs zur Reform verstanden werden. Die hier anzutreffende Kopplung von Kirche, Staat und Gesellschaft ist nämlich das Resultat verschiedenster Formen der Sozialdisziplinierung, etwa der auf Arbeitsbefähigung abzielenden Armengesetze, der Karnevalverbote, der Bürokratisierung verschiedenster Verwaltungsebenen sowie der absolutistischen Steigerung ökonomischer Produktivität, moralischer Konformität und konfessioneller Orthodoxie (S. 108-112), die aus der geschichtswissenschaftlichen und historisch-soziologischen Literatur zum konfessionellen Zeitalter hinlänglich bekannt sind (vgl. nur Gorski 2003). Um der Tatsache Rechnung zu tragen, dass auf dieser Stufe die Gesellschaft noch als organisch eingebettet in eine vor allem in der Lokalgemeinde erfahrbare kirchliche Ordnung imaginiert wird, bezeichnet Taylor sie in etwas eigenwilliger Terminologie auch als ,,paläo-Durkheimianisch“"9: „The ,paleo’ phase corresponds to a situation in which a sense of the ontic dependence of the state on God and higher times is still alive, even though it may be weakened by disenchantment and an instrumental spirit" (S. 455).

Eine zweite Stufe sieht Taylor im 19. Jahrhundert mit der Epoche der Mobilisierung (S. 445) erreicht, in der größere Bevölkerungsgruppen durch Industrialisierung und Urbanisierung aus ihren lokalen Bezügen entbettet, in neue soziale Strukturen eingebunden und für die modernen sozialen Imaginationen empfänglich würden. Die Erfahrung neuer Praktiken in den sozialen Domänen der Ökonomie, der demokratischen Herrschaft und

9 Auf die Probleme dieser Terminologie, die Taylor bereits in seiner Studie zu William James (Taylor 2002) verwendet, hat umsichtig Hans Joas (2004, S. 105) hingewiesen. Zu ergänzen wäre, dass Taylor Durkheims Kirchenbegriff grundlegend missversteht, da dieser gerade nicht mit Blick auf eine begriffliche Scheidung von Kirche und Staat, sondern mit Blick auf den moralischen Gemeinschaftscharakter von Religion formuliert ist vgl. dazu ausführlich Tyrell (2008). 
des öffentlichen Raums hätten den in modernen Theorien moralischer Ordnung artikulierten Annahmen individueller Freiheit, Gleichheit und gegenseitiger Wohltätigkeit Plausibilität verliehen. Wichtig für Taylor ist nun, dass der somit gestärkte immanente Rahmen (zunächst) nicht zu einem Rückgang des Glaubens führte, sondern vielmehr neue religiöse Sozialformen freisetzte. Vor allem zwei Typen unterscheidet er hier. Zunächst die „civil religion“, wie Robert Bellah (1967) sie in Repräsentationen der amerikanischen Nation identifiziert hat, die formal aber auch die viktorianische Gleichsetzung von Christentum und Zivilisation bestimmt, deren Bedeutung für die imperiale Frühphase von Globalisierung bekannt ist (vgl. nur van der Veer 2001). Sodann das religiöse Verbandswesen, wie es die historische Forschung zur evangelikalen Bewegung sowie, in Europa, zu konfessionellen Milieus und nicht zuletzt auch zur katholischen Restauration beleuchtet hat. In beiden Varianten wird (politische oder kollektive) Identität in religiöser Sprache artikuliert, und aus diesem Grund bezeichnet Taylor sie als neo-durkheimianisch: „[I]n ,neo’ societies, God is present because it is his Design around which society is organized. (...). $[\mathrm{T}]$ he senses of belonging to group and confession are fused, and the moral issues of the group's history tend to be coded in religious categories“" (S. 455). Der ohnehin nur allmähliche und ungleichmäßige Verfall älterer Religionsformen sei also, entgegen linearen Säkularisierungstheorien, zunächst von intensiver und gerade auch öffentlich virulenter religiöser Mobilisierung begleitet.

Dies ändert sich in der Epoche der Authentizität, der dritten Station, die Taylor mit der kulturellen Revolution der 1960er Jahre einsetzen lässt und unter das Signum des expressiven Individualismus stellt (S. 473). ${ }^{10}$ Die drei für moderne Theorien moralischer Ordnung besonders resonanzfähigen Domänen von Markt, Demokratie und Öffentlichkeit würden hier durch eine vierte Domäne, nämlich den Bereich der Konsumkultur (S. 481), ergänzt, deren soziale Praktiken nicht den Charakter kollektiven Handelns, sondern gegenseitiger Selbstrepräsentation („mutual display“) hätten. Die zuvor (in der Moderne!) entwickelten öffentlichen Sozialformen der Religion, ob Zivilreligion oder Denomination, würden von einem Vorgang der De-Institutionalisierung erfasst, dessen Folge Entkirchlichung ebenso wie die Entstehung ,post-Durkheimianischer“, individualisierter Religionsformen sei. Entscheidend für Taylor ist, dass die Fragmentierung der Optionen des Glauben und Unglaubens von einem Eliten- zu einem Massenphänomen wird. Diese Optionen reduzierten sich keineswegs auf orthodox religiöse und orthodox säkulare Haltungen, vielmehr entstünden verschiedenste, zwischen Generationen sowie innerhalb einzelner Lebensläufe wechselnde Aneignungen der religiösen Tradition und individuelle Spiritualitätsformen, die allesamt, und damit erreicht Taylor die Pointe seiner historischen Rekonstruktion, auch als Optionen erfahren würden.

10 Das Ideal der Authentizität hatte Taylor bereits in Quellen des Selbst auf seine historischen Quellen in der Romantik untersucht und in seiner moralischen Ambivalenz ausgeleuchtet; vgl. Taylor (1995 [1991], S. 32). Hier wie dort erstaunt, wie wenig Aufmerksamkeit Taylor den Erfahrungen des Imperialismus sowie der beiden Weltkriege schenkt, die doch in der ersten Hälfte des 20. Jahrhunderts Anlass für verschiedenste religiöse Bewegungen waren. 


\subsection{Ein neuer Blick auf die moderne Religionsgeschichte?}

Taylors kulturalistische Säkularitätserzählung geht sowohl in der Breite als auch im Detailreichtum des historischen Materials über konventionelle Säkularisierungstheorien hinaus. Sie zeichnet ein Bild der modernen Religionsgeschichte, das deren verstrickte und teils gegenläufige Entwicklungen einzufangen und damit dem zweiten der eingangs genannten Ansprüche Rechnung zu tragen vermag. Gewiss, unschwer lassen sich auch Lücken in Taylors Erzählung identifizieren. Insbesondere ist man verwundert, dass die begriffsgeschichtlich bestens erschlossene Neuerfindung von „Religion“, die im 17. Jahrhundert einsetzt und im 19. Jahrhundert mit der historisch-kritischen Erforschung von „Weltreligionen“ ihren vorläufigen Abschluss findet (vgl. Asad 1993; Masuzawa 2005), nicht weiter reflektiert wird. Dies wäre deshalb wichtig, weil im modernen Religionsbegriff selbst sich die Entstehung des immanenten Rahmens in nuce widerspiegelt (vgl. Tenbruck 1993). Ebenso irritiert (zumindest den deutschsprachigen Leser), dass Taylor die Schmitt-Blumenberg-Debatte um das Verhältnis von Christentum und Moderne nicht weiter diskutiert (vgl. nur Monod 2002). Tatsächlich ist Säkularisierung seit dem 19. Jahrhundert ja nicht nur nur als Subtraktions- oder Diskontinuitäts-, sondern stets auch als Kontinuitätsgeschichte erzählt worden. Da Taylor selbst auf die christlichen Ursprünge eines sich selbst missverstehenden immanenten Rahmens abhebt, wäre eine Auseinandersetzung mit den diesbezüglichen Einwänden von Blumenberg (1988), den Taylor etwas lapidar als Subtraktionserzähler einordnet (S. 294), geboten gewesen.

Doch trotz solcher Einwände beeindruckt Taylors kulturtheoretische Rekonstruktion der Entstehung und Ausbreitung des immanenten Rahmens darin, wie sie die empirischen Evidenzen für einen Rückgang religiösen Glaubens (,Säkularität 2“), die stets den Eindruck der Irreversibilität von Säkularisierung erzeugt hatten, mit Befunden religiöser Revitalisierung verbindet. Entkirchlichungsprozesse lassen sich damit als Episoden einer höchst bewegten Religionsgeschichte der Moderne begreifen, in der sich Phasen religiöser Mobilisierung und Phasen religiösen Niedergangs in länder- und regionalspezifischer Weise abwechseln. Die religiösen Erneuerungsbewegungen im Amerika des 19. Jahrhunderts (Finke und Stark 2005; Thomas 1996) ebenso wie die Milieubildungen im „Zweiten konfessionellen Zeitalter“ in Europa (Blaschke 2000) lassen sich als spezifisch moderne, d. h. den immanenten Rahmen voraussetzende Religionsformen deuten. Und auch die Inanspruchnahme von Religion für die Konstruktion ethnischer, nationaler oder zivilisatorischer Identitäten muss nicht mehr residual als kulturelle Abwehr konzipiert werden (Bruce 2002, S. 31), sondern gliedert sich, wie jene Identitäten selbst, in die Welt moderner sozialer Imaginationen ein. Taylors Darstellung des Zeitalters der Authentizität, also des Zeitfensters, für das die religionssoziologische Survey-Forschung in Europa dramatische Entkirchlichungsprozesse konstantiert, trifft sich mit den revisionistischen Historiographien von Hugh McLeod (2007) und Callum Brown (2001), ohne dabei den Blick für die massenkulturellen Formen individualisierter Religion zu verlieren (vgl. Davie 2000; Hervieu-Léger 2004). Unschwer ließen sich auf Taylors Linie aber auch globale fundamentalistische Bewegungen deuten, nämlich als kulturell entbettete Sozialformen von Religion und somit gerade als Folge der Ausbreitung des immanenten Rahmens (vgl. dazu Eisenstadt 1998). Lediglich Formen religiös indifferenter Lebensführung (vgl. Wohlrab-Sahr et al. 2009, S. 263 ff.; Zuckermann 2009) müssen in Taylors Erzählung 
unterbelichtet bleiben, weil seine formale Anthropologie außeralltägliche Erfahrungen von Erfüllung, seien sie transzendent oder immanent, analytisch privilegiert (vgl. Butler in Warner et al. 2010, S. 206 ff.).

Dass Taylor eine weitgehend schlüssige Erzählung der Religionsgeschichte der Moderne bietet, besagt natürlich noch nicht, dass nicht bessere Alternativentwürfe zur konventionellen Säkularisierungstheorie denkbar wären. Auch systemtheoretische Entwürfe böten schließlich eine auf den ersten Blick nicht unplausible, nämlich differenzierungstheoretische Erklärung dafür, dass Religion in der Moderne an gesellschaftsstrukturellem Rückhalt verliert, aber auf diese Situation mit (organisations- und bewegungsförmigen) Neubildungen auch reagieren kann (Luhmann 2000, S. 285). Damit aber stellt sich die Frage, wie sich Taylors kulturalistische Rekonstruktion des immanenten Rahmens in der Kontroverse um die Differenzierung religiöser und anderen gesellschaftlichen Ordnungen positioniert.

\section{Der immanente Rahmen und die Kontroverse um Differenzierung}

Dass ein Alternativentwurf zum konventionellen Säkularisierungsparadigma den Variantenreichtum der Differenzierungsmuster moderner Gesellschaften beschreiben und erklären können sollte, wurde eingangs als dritter Anspruch formuliert. Denn auch die Differenzierungsthese, lange der unbestrittene paradigmatische Kern des Säkularisierungsparadigmas, sieht sich gegenwärtig harter Kritik ausgesetzt. Gegenüber unilinearen Konzeptionen zunehmender Differenzierung von Religion und Politik macht die von Shmuel N. Eisenstadt angestoßene Debatte um multiple Modernitäten (vgl. überblickshalber Schwinn 2009) in deskriptiver Hinsicht auf die Variabilität von Differenzierungsmusters aufmerksam (so schon Martin 1978; neuerdings z. B. Gorski 2000, 2005; Katznelson und Jones 2010; Spohn 2008). Und in explanatorischer Hinsicht steht das Problem auf der Tagesordnung, wie die Differenzierung von religiöser und politischer Ordnung allgemein und in ihren spezifischen Ausprägungen zu erklären ist. ${ }^{11}$ Mit Blick auf Taylors Alternativentwurf ist daher zu diskutieren, welchen Beitrag die Rekonstruktion des immanenten Rahmens (,Säkularität 3“) für die Erklärung der variantenreichen Differenzierungsmuster von Religion und Politik (,Säkularität 1“) leistet.

Diesbezüglich bleibt Taylor indessen hinter den zu stellenden Ansprüchen zurück, und zwar aus zwei systematischen Gründen. Erstens bleibt seine kulturalistische Theorie moderner Säkularität auf dem Weg zu einer kulturvergleichenden Soziologie multipler Säkularitäten auf halbem Wege stecken. Und zweitens trägt sie nur wenig zur Klärung der kausalen Mechanismen bei, die den Schüben der Differenzierung von politischer und religiöser Ordnung und ihren verschiedenen institutionellen Ergebnissen zu Grunde liegen.

11 Dies gilt im Übrigen nicht nur für die religionssoziologische Säkularisierungsdebatte, sondern auch für die allgemeine soziologische Theorie; vgl. nur Schwinn (2001, S. 25). 


\subsection{Kulturelle Prämissen von Differenzierung}

Zunächst ist zu betonen, dass Taylors Rekonstruktion des immanenten Rahmens durchaus zum besseren Verständnis der Differenzierung von Religion und Politik in der westlichen Moderne beitragen kann. Überzeugend erläutert Taylor, dass die Entstehung autonomer öffentlicher Sphären, nationaler Gemeinschaften und souveräner Staaten als notwendige Bedingung die Imagination einer von der Heilsgeschichte getrennten historischen Zeit und eines aus kirchlichen Hierarchien gelösten und direkt zugänglichen Sozialraums voraussetzte - und dass diese ein Nebenprodukt des christlichen Drangs zur Reform waren. Damit aber leuchtet er den kulturellen Horizont sich wechselseitig stabilisierender Weltvorstellungen aus, der eine schärfere Differenzierung politischer von religiöser Ordnungsbildung überhaupt erst hat möglich werden lassen, und erfasst gleichzeitigt die fortdauernde kulturelle Imprägnierung der Inklusionsmuster differenzierter Ordnungen durch christliche Werte. ${ }^{12}$

Eine solche kulturalistische Rekonstruktion der Voraussetzungen von Differenzierung, auf ihre theoretischen Probleme wird unten einzugehen sein, provoziert allerdings geradezu den Vergleich zu außereuropäischen Kulturen. Nur er würde es schließlich gestatten, die Besonderheiten des Christentums kenntlich zu machen, die den Westen zum Schauplatz der Entstehung des immanenten Rahmens und entsprechender Differenzierungsprozesse gemacht haben. Den Blick über den westlichen Kulturraum wagt Taylor indessen ausdrücklich nicht. Als Grund hierfür nennt er nicht nur die zu bewältigende Materialfülle, sondern vor allem seine hermeneutischen Intentionen, die auf Selbstvergewisserung der Quellen des Sinns in der europäischen Moderne (im Text als „we“ markiert) zielt. Genau an dieser Stelle zeigt sich indessen eine gewisse Spannung zwischen Taylors hermeneutischen Intentionen und seinem kulturalistischen Theoriedesign. Einerseits schränkt er die Bestimmung religiösen Glaubens als eine an Transzendenz und menschlicher Transformation orientierte Variante der Deutung von Erfüllungserfahrungen (s. o.) auf den zivilisatorischen Raum des westlichen Christentums ein (S. 15). Andererseits verwendet er diesen Religionsbegriff jedoch in einem sehr viel allgemeineren und vergleichsaffinen Sinne, wenn er behauptet dass alle Religionen der Achsenzeit, also Konfuzianismus, Hinduismus, Buddhismus sowie die monotheistischen Erlösungsreligionen durch Radikalisierung von Transzendenz neue Möglichkeiten menschlicher Transformation eröffnet hätten (S. 151 ff., S. 611).

Tatsächlich folgt Taylor streckenweise unmittelbar den Überlegungen von Eisenstadt (1982; 2003; vgl. auch Bellah 2005), der das Konzept der Axialität, verstanden als Spannung zwischen transzendenter und mundaner Sphäre, zwischen „Welt““ und „Hinterwelt", aus der heraus gegebene Ordnungen als rekonstruktionsbedürftig erscheinen, in die komparative Makrosoziologie eingeführt hat. Auch Taylors Ausführungen zu den gesellschaftsstrukturellen Korrelaten von Axialität, insbesondere die Entstehung von religiösem Virtuosentum, das in Spannung zur weiterhin vor-axial geprägten Massenreligiosität steht, schließen hier an. Das Konzept der Achsenzeitreligionen übernimmt sogar eine tragende Rolle in Taylors Erzählung von der Genese des immanenten Rahmens. Denn für die christliche Achsenzeit macht er geltend, dass die Spannung zwischen Virtuosen- und

12 Ähnliche Überlegungen finden sich bei Parsons (1967, S. 391) sowie bei Schwinn (2001). 
Massenreligiosität zunehmend als unbefriedigend erfahren worden sei (S. 155). In den Versuchen ihrer Überwindung liege der Ursprung des Drangs zur Reform, der seinerseits, wie oben gesehen, den Auftakt für die Konstruktion des immanenten Rahmens darstellt. Wenn der Durchbruch zum säkularen Zeitalter allerdings in einer achsenzeitlichen Dynamik zu verorten ist, wäre zu klären, ob und warum er nur im Rahmen der christlichen Achsenzeit erfolgte. Dass man diese Frage ohne interkulturelle Vergleiche nicht beantworten kann, liegt auf der Hand.

Noch wichtiger werden interkulturelle Vergleiche, wenn man nicht nur historisch nach der Konstruktion des immanenten Rahmens und entsprechender Differenzierungsmuster, sondern auch nach deren gegenwärtigen Varianten fragt. Genau auf diese Frage zielt letztlich Eisenstadts Konzeption multipler Modernität, wonach aus dem Zusammentreffen des kulturellen Programms der westlichen Moderne mit anderen Varianten der axialen Spannung zwischen transzendenter und immanenter Sphäre unterschiedliche Ordnungsmuster hervorgegangen seien. Auch für Taylor läge es nahe, diese Frage zu traktieren. Denn wiewohl er den Drang zur Reform und die ihr folgende Institutionalisierung des immanenten Rahmens zunächst noch als singulär christliches Ereignis deutet, behauptet er, dass die sozialen Imaginationen der Moderne dank ihres inhärenten Universalismus heute eine globale Ausbreitung erfahren hätten (Taylor 2004). Diese weltgesellschaftliche Ausdehnung des immanenten Rahmens aber kann, wie Casanova (2009, S. 98, 104) treffend argumentiert hat, beim Zusammentreffen mit anderen axialen Zivilisationen spezifische Varianten des immanenten Rahmens (,Säkularität 3“) erzeugen, die des europäischen Stadienbewusstseins entbehren und mithin gänzlich andere Differenzierungsmuster (,Säkularität $1^{\text {“) }}$ ) erzeugen (vgl. dazu auch Koenig 2007). Deren komparative Analyse bleibt bei Taylor trotz vereinzelter Andeutungen jedoch ebenso Desideratum wie das Bewusstsein für die verschiedenen, zumal die kolonialen inter-zivilisatorischen Begegnungen, die ihrerseits den okzidentalen Säkularisierungspfad nachhaltig geprägt haben. ${ }^{13}$

$\mathrm{Ob}$ sich die Spannung zwischen Taylors hermeneutischen Intentionen und seiner Inanspruchnahme von Eisenstadts Theorie der Achsenzeitkulturen auflösen lässt, muss hier nicht abschließend entschieden werden. ${ }^{14}$ Offen kann auch bleiben, inwieweit das Achsenzeitkonzept und eine darauf aufbauende komparative Zivilisationsanalyse als solche überhaupt tragfähig sind (kritisch Breuer 1994; Knöbl 2007). Nachgewiesen werden sollte lediglich, dass eine kulturalistische Theorie moderner Säkularität, ihrer okzidentalen Ursprünge und ihrer globaler Diffusion ohne kulturvergleichende und transfergeschichtliche Analysen defizitär bleibt. Für die religionssoziologische Forschung, die über das konventionelle Säkularisierungsparadigma hinausgehen möchte, liegt hier jedenfalls eine Fülle zu klärender historischer und empirischer Fragen.

13 Nur in seiner politisch-philosophischen Analyse von „Säkularität 2“ greift Taylor am Beispiel Indiens über den christlichen Kulturhorizont hinaus; vgl. Taylor (1996, S. 218, 236 ff.) Zur Bedeutung kolonialer Konstellationen für den europäischen Säkularisierungspfad vgl. van der Veer (2001).

14 Es besteht diesbezüglich durchaus Grund zur Skepsis, ist doch die Theorie der Achsenzeitkulturen ein spätes Erbe der Erfindung von „Weltreligionen“ (Masuzawa 2005) und setzt mithin eine relativierende Perspektive auf Religion als „Kultur“ (Luhmann 2000) voraus, womit die Würfel bereits zugunsten der Naturalisierung des immanenten Rahmens gefallen sind. 


\subsection{Imaginationen, Institutionen und Interessen}

Damit aber sind schließlich die grundlegenden Probleme einer kulturalistischen Theorie moderner Säkularität anzusprechen. So sehr sie zur Rekonstruktion der Voraussetzungen von Differenzierung beiträgt, so wenig vermag sie die spezifischen Differenzierungsmuster zu erklären, für die sich die komparative Forschung zum Verhältnis von religiöser und politischer Ordnung gegenwärtig interessiert. Schon hinsichtlich der Beschreibung unterschiedlicher Differenzierungsmuster bleibt Taylors Darstellung des säkularen Zeitalters recht blass, wenn man sie mit dem Auflösungsniveau gegenwärtiger Typologien und Kategorisierungen kontrastiert (vgl. nur Fox 2008; Kuru 2009). Nicht einmal die in seinen politisch-philosophischen Schriften (Taylor 1996) entwickelte Unterscheidung dreier Modelle von „Säkularismus“ - die „Strategie des gemeinsamen Fundamentes“ (Pufendorf), die „Strategie der unabhängigen politischen Ethik“ (Hobbes) und die „Strategie eines übergreifenden Konsens" (Rawls) - macht Taylor komparativ fruchtbar. Soweit er Verhältnisse religiöser und politischer Ordnung überhaupt in den Blick nimmt, so unter Rückgriff auf die ohnedies fraglichen paläo-, neo- und post-Durkheimschen Konfigurationen; transatlantische Unterschiede werden dabei in Tocqueville'scher Manier auf die unterschiedlichen Situationen zurückgeführt, in denen moderne soziale Imaginationen wirksam wurden. Gingen sie in der Französischen Revolution mit der Erfindung neuer Praktiken einher, führten sie in der Amerikanischen Revolution in Ermangelung einer „paläo-Durkheim'schen“ Phase zur Re-Interpretation schon bestehender sozialer Praktiken (vgl. ähnlich Casanova 2009, S. 102; Berger et al. 2008).

Erst recht aber bleibt Taylor hinsichtlich der Erklärung unterschiedlicher Differenzierungsmuster hinter Entwicklungen in der historischen Soziologie zurück, die das Problem wirksamer kausaler Mechanismen ins Zentrum der Kontroverse um das Differenzierungstheorem gerückt haben (vgl. Gorski 2000; Smith 2003). Gerade hier zeigt sich, dass Taylors kulturalistische Säkularisierungserzählung an den theoretischen Problemen und methodischen Fallstricken krankt, denen streng kulturalistische Theorien der Moderne generell ausgesetzt sind. Dieser Probleme ist sich Taylor durchaus bewusst. Offen setzt er sich mit dem Einwand auseinander, seine narrative Rekonstruktion moderner Imaginationen leide an einer idealistischen Schlagseite. Zu entkräften versucht er ihn mit zwei Argumenten. Das eine besagt, der Einwand beruhe letztlich auf einer falschen Dichotomie von ideellen und materiellen Faktoren (Taylor 2004, S. 31). Soziale Praktiken seien stets gleichermaßen von ideellen wie materiellen Dimensionen durchdrungen. Hintergrund (im Wittgenstein'schen Sinne) und Praktiken stünden in einem wechselseitigen Verhältnis zueinander; soziale Praktiken würden durch einen gemeinsamen Hintergrund erst ermöglicht, dieser sei seinerseits aber nur in jenen gegenwärtig (S. 212 f.). Das andere Argument besteht in einer Zurückweisung der Ansprüche an starke Kausalerklärungen schlechthin. Die Rekonstruktion der „für uns“ zugänglichen Welthaltungen ziele gar nicht auf historisch-soziologische Erklärung, sondern auf hermeneutische Verständigung (Taylor 1995, S. 354 f.). Gänzlich überzeugen können diese Verteidigungsversuche indessen nicht. Auch eine narrative Rekonstruktion mit vorwiegend hermeneutischen Absichten kann sich den Plausibilitätsansprüchen historischer Erklärungen nicht verweigern (vgl. Smith 2002, S. 213). Und gerade wenn man den ideell-materiellen Doppelcharakter sozialer Praktiken in Rechnung stellt, bleibt eine idealistische top-down-Erklärung, die jegliche 
Macht- und Interessenkonstellationen ausblendet, ergänzungsbedürftig (vgl. Rosa 1998, S. 282).

Die neuere historisch-soziologische Debatte, die sich der explanatorischen Vertiefung des Differenzierungstheorems widmet, rückt neben kulturellen daher auch andere Faktoren, insbesondere Interessen- und Machtkonstellationen, in den Vordergrund. Eine dezidiert anti-kulturalistische Position bezieht beispielsweise Anthony Gill (2008), der am Beispiel der USA, Lateinamerikas und der post-sowjetischen Staaten ein theoretisches Modell der Entstehung von Religionsfreiheit, also einer spezifischen Komponente der Differenzierung politischer und religiöser Ordnung entwickelt hat. Das Modell geht axiomatisch von typischen Interessen politischer Machtinhaber (an Machterhalt), religiöser Mehrheiten (an Regulierung des religiösen Feldes) und religiöser Minderheiten (an Deregulierung des religiösen Feldes) aus und benennt Bedingungen auf politischer Seite (stabile Machtperspektive und hohe Opportunitätskosten von Regulierung) sowie auf religiöser Seite (Pluralisierung), unter denen, bei Unterstellung rationaler Handlungswahlen aller drei Akteursgruppen, die Gewährleistung von Religionsfreiheit oder allgemein die Deregulierung des religiösen Feldes wahrscheinlich wird. Erklärungskraft besitzt ein interessenbasiertes Modell nicht nur, weil es situative Bedingungen für die politische Gewähr von Religionsfreiheit benennt, sondern auch weil es soziale Mechanismen sich selbst steigernder Differenzierung identifiziert. Hat Pluralisierung, wie etwa im amerikanischen Fall, erst einmal einen kritischen Schwellenwert erreicht, erhöht sie die Opportunitätskosten restriktiver Religionspolitik mit dem Resultat schrittweiser Deregulierung des religiösen Feldes, die ihrerseits weitere Pluralisierung freisetzt, und so fort. Gewiss, ein rein interessenbasiertes Modell bleibt darin unvollständig, dass es die Identitäten und Präferenzen der Akteure als exogen gegeben annimmt, statt sie selbst zum Gegenstand historischer Erklärung zu machen (vgl. Katzelnson und Jones 2010, S. 65). Unvollständig bleibt das Modell auch darin, dass es ungeklärt lässt, unter welchen Bedingungen Religionsfreiheit auch gegen widrige Interessenkonstellationen auf Dauer gestellt, also als einklagbares subjektives Recht institutionalisiert werden kann. Dass die Erklärung der Genese spezifischer Differenzierungsmuster und ihrer Institutionalisierung ohne eine Analyse der Interessenlagen politischer und religiöser Akteure defizitär bleiben muss, wird man aber ebenfalls zugestehen müssen (vgl. Chaves 1994; Smith 2003). Und auch die Reproduktion von Differenzierungsmustern wird man im Lichte der Diskussion um Mechanismen historischer Pfadabhängigkeit auf die Machtkonstellationen ihrer Trägergruppen hin befragen müssen (vgl. Kuru 2009).

Wenn man aber diese Probleme der Erklärung von Varianten institutioneller Differenzierung (,Säkularität 1“) ernst nimmt, gerät Taylors kulturalistische Säkularitätsnarrativ auch dort ins Wanken, wo es um die Erklärung der sozialen Imaginationen selbst (,Säkularität 3") geht. Auf beiden Ebenen seiner Rekonstruktion blendet Taylor nämlich das Konfliktgeschehen aus, das die Entstehung des immanenten Rahmens begleitet hat. Dies gilt zunächst für die Formulierung neuer Theorien von Welt, Gesellschaft und Selbst. So lässt sich die Dynamik kultureller Kreativität nicht verstehen, wenn man nicht auch die inneren Konflikte des intellektuellen Feldes berücksichtigt, das an Autonomie gewonnen hatte, nachdem die katholischen und protestantischen Kirchen als Resultat der Konfessionskonflikte im 16. Jahrhundert die Kontrolle über die Mittel intellektueller Produktion an die aufsteigenden Territorialstaaten verloren hatten (vgl. Collins 1998, S. 570-617; 
vgl. auch Wuthnow 1989). Noch weniger geht Taylor auf das Konfliktgeschehen ein, das den Übergang moderner Ordnungstheorien in die sozialen Imaginationen ganzer Bevölkerungen begleitete. Dass die Entstehung einer säkularen Hegemonialkultur das Ergebnis heftiger Machtkämpfe zwischen protestantischen Eliten und aufstrebenden Professionsgruppen in Bildung, Medizin, Recht, Medien und Wissenschaft war, in deren Folge die institutionelle Kontrolle der Kirche über kulturelle Produktion allgemein eingeschränkt wurde, hat beispielsweise die Autorengruppe um Christian Smith (2003) am Beispiel der amerikanischen Religionsgeschichte des späten 19. Jahrhunderts materialreich dargestellt. Und auch die Geschichte der Kulturkämpfe in Europa zeigt, dass die Hegemonie säkularer Imaginationen Resultat heftiger, teils gewaltförmiger Konflikte war (Poulat 1987; Clark und Kaiser 2003; Borutta 2010). Auch für die historisch-komparative Analyse der Varianten von ,Säkularität 3“ sind mithin Interessenlagen und institutionelle Kontexte stärker in Rechnung zu stellen, als Taylors kulturalistischer Zugriff es gestattet.

\section{Fazit - einige Aufgaben historisch-soziologischer Religionssoziologie}

Taylors monumentale Studie A Secular Age ist zweifellos ein bedeutender Beitrag zur aktuellen Debatte um Religion in der Moderne. Gegenüber zeitdiagnostischen Prognosen „,post-säkularer" Gesellschaften unterstreicht sie, wie wenig gewonnen wäre, wollte man die Säkularität der Moderne bestreiten. Gleichzeitig lässt sich an ihr studieren, mit welchen Problemen die Formulierung eines Alternativentwurfs zur konventionellen Säkularisierungstheorie konfrontiert ist.

Unter den eingangs genannten Ansprüchen erfüllt sie den ersten in ganz offenkundiger Weise, zielt Taylors gesamtes Unternehmen doch auf eine Rekonstruktion des säkularen Selbstverständnisses der (westlichen) Moderne. Nicht zuletzt gegenüber den Sozialwissenschaften, die aufs Engste in die Konstruktion des immanenten Rahmens verstrickt waren, nimmt Taylors formalanthropologisch fundierte Kulturtheorie des säkularen Zeitalters eine reflexive Perspektive ein. Sie unterstreicht nicht nur die Berechtigung der Forschung zu den Genealogien sowohl von Diskontinuitäts- als auch Kontinuitätsnarrativen der Säkularisierung (vgl. Borutta 2010), sondern fordert auch zu erneuterter Klärung der Hintergrundannahmen soziologischer Religionstheorie heraus. Taylor gelingt es zweitens, einen Deutungsrahmen aufzuspannen, innerhalb dessen sich vermeintlich widersprüchliche Entwicklungen von Religion in der Moderne integrieren lassen. Die kulturelle Konstruktion des immanenten Rahmens ermöglicht Entkirchlichungsprozesse, wie sie von klassischen Säkularisierungstheoretikern unterstrichen wurden (,Säkularität 2“), setzt aber auch die kollektive und individuelle Erfindung neuer religiöser Identitäten frei. Eine weitere Aufgabe religionssoziologischer Forschung liegt mithin darin, die genauen Bedingungen zu bestimmen, unter denen der immanente Rahmen die eine oder andere Wirkung entfaltet. Von allgemeinen Trendaussagen zur Analyse kausaler Mechanismen vorzudringen, ist auch die bleibende Aufgabe komparativer Forschung zu Muster der Differenzierung religiöser und politischer Ordnung (,Säkularität 1“). Gerade in kultur- und gesellschaftsvergleichender Hinsicht kann Taylors kulturalistische Erzählung der Säkularisierung nämlich, drittens, nicht alle Wünsche befriedigen. Sie ist arm an analytischen Instrumenten, um das Konfliktgeschehen sowie die ihm zu Grunde liegenden Interessen- 
und Machtkonstellationen zu erfassen, aus denen nicht nur institutionelle Arrangements von Religion und Politik, sondern auch die sozialen Imaginationen selbst hervorgehen.

Doch auch wenn Taylors Alternativentwurf zum konventionellen Säkularisierungsparadgima viele Antworten schuldig bleibt, wirft er reichhaltige Fragen auf, denen sich die (historisch-) soziologische Forschung widmen müsste - Fragen, die allesamt von großen Erzählungen weg- und zu kausalanalytischen Studien hinführen. Ob eine solche geschichtsphilosophisch und zeitdiagnostisch bescheidenere Agenda Taylors eigenem Anspruch einer hermeneutischen Vermittlung der Binnenperspektiven verschiedener Erfahrungen von Erfüllung gerecht werden kann, mag man bezweifeln. Es darf aber durchaus gehofft werden, dass sie der Aufklärung gegenwärtiger Religionskontroversen zugute käme.

Open Access: Dieser Artikel unterliegt den Bedingungen der Creative Commons Attribution Noncommercial License. Dadurch sind die nichtkommerzielle Nutzung, Verteilung und Reproduktion erlaubt, sofern der/die Originalautor/en und die Quelle angegeben sind.

\section{Literatur}

Alexander, Jeffrey C. 2003. The meanings of social life: A cultural sociology. Oxford: Oxford University Press.

Asad, Talal. 1993. Genealogies of religion. Discipline and reasons of power in Christianity and Islam. Baltimore: Johns Hopkins University Press.

Asad, Talal. 1999. Religion, nation-state, secularism. In Nation and religion: Perspectives on Europe and Asia, Hrsg. Peter van der Veer und Hartmut Lehmann, 178-196. Princeton: Princeton University Press.

Asad, Talal. 2003. Formations of the secular. Christianity, Islam, Modernity. Stanford: Stanford University Press.

Ausmus, Harry J. 1982. The polite escape. On the myth of secularization. Athens: Ohio University Press.

Bellah, Robert N. 1967. Religion in America. Daedalus. Journal of the American Academy of Arts and Science 96:1-21.

Bellah, Robert N. 2005. What is axial about the Axial Age? Archives européennes de sociologie 46:69-87.

Berger, Peter L. 1973. Zur Dialektik von Religion und Gesellschaft. Elemente einer soziologischen Theorie. Frankfurt a. M.: Fischer. (1967).

Berger, Peter L. 1992. Der Zwang zur Häresie. Religion in der pluralistischen Gesellschaft. Freiburg: Herder. (1980).

Berger, Peter L. (Hrsg.). 1999. The desecularization of the world. Resurgent religion and world politics. Washington: Ethics and Public Policy Center.

Berger, Peter L., Grace Davie, und Effie Fokas. 2008. Religious America, secular Europe? A theme and variations. Aldershot: Ashgate.

Berman, Harold J. 1983. Law and revolution. The formation of the Western legal tradition. Cambridge: Harvard University Press.

Berman, Harold J. 2004. Law and revolution II. The impact of the Protestant reformations on the Western legal tradition. Harvard: Harvard University Press.

Blaschke, Olaf. 2000. Das 19. Jahrhundert: Ein zweites konfessionelles Zeitalter? Geschichte und Gesellschaft 26:38-75. 
Blumenberg, Hans. 1988. Die Legitimität der Neuzeit (1966). Frankfurt a. M.: Suhrkamp.

Borutta, Manuel. 2010. Genealogie der Säkularisierungstheorie. Zur Historisierung einer großen Erzählung der Moderne. Geschichte und Gesellschaft 36:347-376.

Breuer, Stefan. 1994. Kulturen der Achsenzeit. Leistung und Grenzen eines geschichtsphilosophischen Konzepts. Saeculum 45:1-33.

Brown, Callum. 2001. The death of Christian Britain. London: Routledge.

Bruce, Steve. 1996. Religion in the modern world. From cathedrals to cults. Oxford: Oxford University Press.

Bruce, Steve. 2002. God is dead. Secularization in the west. Oxford: Blackwell.

Calhoun, Craig. 2008. A secular age, archives européennes de sociologie 49:455-461.

Casanova, José. 1994. Public religions in the modern world. Chicago: Chicago University Press.

Casanova, José. 2006. Rethinking secularization: A global comparative perspectives. The Hedgehog Review 8:7-22.

Casanova, José. 2008. Public religions revisited. In Religion: Beyond the concept, Hrsg. Hent de Vries, 101-119. Fordham: Fordham University Press.

Casanova, José. 2009. Europas Angst vor der Religion. Berlin: Berlin University Press.

Chaves, Mark. 1994. Secularization as declining religious authority. Social Forces 72:749-774.

Clark, Christopher, und Wolfram Kaiser (Hrsg.). 2003. Culture wars. Secular-catholic conflict in nineteenth century Europe. Cambridge: Cambridge University Press.

Collins, Randall. 1998. The sociology of philosophies. A global theory of intellectual change. Cambridge: Harvard University Press.

Davie, Grace. 2000. Religion in modern Europe. A memory mutates. Oxford: Oxford University Press.

Dobbelaere, Karel. 1981. Secularization. A multi-dimensional concept. London: Sage.

Ebaugh, Helen R. 2002. Return of the sacred: Reintegrating religion in the social sciences. Journal for the Scientific Study of Religion 41:385-395.

Eisenstadt, Shmuel N. 1982. The axial age: The emergence of transcendental visions and the rise of the clerics. Archives européennes de sociologie 23:299-314.

Eisenstadt, Shmuel N. 1998. Die Antinomien der Moderne. Die jakobinischen Grundzüge der Moderne und des Fundamentalismus. Heterodoxien, Utopismus und Jakobinismus in der Konstitution fundamentalistischer Bewegungen. Frankfurt a. M.: Suhrkamp.

Eisenstadt, Shmuel N. 2003. Comparative civilizations and multiple modernities. Leiden: Brill.

Finke, Roger, und Rodney Stark. 2005. The churching of America: Winners and losers. New Brunswick: Rutgers University Press.

Fox, Jonathan. 2008. A world survey of religion and the state. Cambridge: Cambridge University Press.

Gauchet, Marcel. 1985. Le désenchantement du monde. Une histoire politique de la religion. Paris: Gallimard.

Gill, Anthony. 2008. The political origins of religious liberty. Cambridge: Cambridge University Press.

Glasner, P. 1977. The sociology of secularization. A critique of a concept. London: Routledge \& Kegan Paul.

Gorski, Philip S. 2000. Historicizing the secularization debate. American Sociological Review 65:138-167.

Gorski, Philip S. 2003. The disciplinary revolution. Calvinism and the rise of the state in early modern Europe. Chicago: University of Chicago Press.

Gorski, Philip S. 2005. The return of the repressed: Religion and the political unconscious of historical sociology. In Remaking modernity. Politics, history, and sociology, Hrsg. Julia Adams, Elisabeth S. Clemens und Ann Shola Orloff, 161-189. Durham: Duke University Press.

Gorski, Philip S., und Ates Altınordu. 2008. After secularization? Annual Review of Sociology 34:55-85. 
Habermas, Jürgen. 1981. Theorie des kommunikativen Handelns. 2 Bde. Frankfurt a. M.: Suhrkamp.

Habermas, Jürgen. 2001. Glauben und Wissen. Rede anläßlich der Verleihung des Friedenspreises des Deutschen Buchhandels. Frankfurt a. M.: Suhrkamp.

Hadden, Jeffrey K. 1987. Toward desacralizing secularization theory. Social Forces 65:587-611.

Hervieu-Léger, Danièle. 2004. Pilger und Konvertiten. Religion in Bewegung. Würzburg: Ergon-Verlag.

Hurd, Elizabeth S. 2004. The political authority of secularism in international relations. European Journal of International Relations 10:235-262.

Hurd, Elizabeth S. 2008. A secular age, by Charles Taylor. Political Theory 36:486-491.

Joas, Hans. 1997. Die Entstehung der Werte. Frankfurt a. M.: Suhrkamp.

Joas, Hans. 2004. Braucht der Mensch Religion? Freiburg: Herder.

Joas, Hans. 2009. Die säkulare Option. Ihr Aufstieg und ihre Folgen. Deutsche Zeitschrift für Philosophie 57:293-300.

Katznelson, Ira, und Gareth Stedman Jones (Hrsg.). 2010. Religion and the political imagination. Cambridge: Cambridge University Press.

Knöbl, Wolfgang. 2007. Die Kontingenz der Moderne. Wege in Europa, Asien und Amerika. Frankfurt a. M.: Campus.

Koenig, Matthias. 2007. Kulturelle Konstruktionen und institutionelle Varianten der Moderne in der Weltgesellschaft. In Kulturen der Moderne. Soziologische Perspektiven der Gegenwart, Hrsg. Andreas Reckwitz und Thorsten Bonacker, 71-96. Frankfurt a. M.: Campus Verlag.

Kühnlein, Michael. 2008. Religion als Quelle des Selbst. Zur Vernunft- und Freiheitskritik von Charles Taylor. Tübingen: Mohr Siebeck.

Kuru, Ahmet T. 2009. Secularism and state policies toward religion. The United States, France, and Turkey. Cambridge: Cambridge University Press.

Lehmann, Hartmut. 2009. Entzauberung der Welt. Studien zu Themen von Max Weber. Göttingen: Wallstein Verlag.

Luckmann, Thomas. 1991. Die unsichtbare Religion. Frankfurt a. M.: Suhrkamp. (1967).

Luckmann, Thomas. 2002. Wissen und Gesellschaft. Ausgewählte Aufsätze 1981-2002. Konstanz: UVK.

Luhmann, Niklas. 2000. Die Religion der Gesellschaft. Frankfurt a. M.: Suhrkamp.

Martin, David. 1965. Towards eliminating the concept of secularization. In Penguin survey of the social sciences, Hrsg. J. Gould, 169-182. Harmondsworth: Penguin.

Martin, David. 1978. A general theory of secularization. Oxford: Basil Blackwell.

Masuzawa, Tomoko. 2005. The invention of world religions. Or, how European universalism was preserved in the language of pluralism. Chicago: Chicago University Press.

McLeod, Hugh. 2007. The religious crisis of the 1960 s. Oxford: Oxford University Press.

McLennan, G. 2008. Among the unbelievers. New Left Review 52:139-148.

Meyer, John W., John Boli, und George M. Thomas. 1987. Ontology and rationalization in the western cultural account. In Institutional structure. constituting state, society, and the individual, Hrsg. George M. Thomas, John W. Meyer und John Boli, 12-37. Newbury Park: Sage Publications.

Milbank, John. 1990. Theology and social theory. Beyond secular reason. Oxford: Blackwell.

Monod, Jean-Claude. 2002. La querelle de la sécularisation de Hegel à Blumenberg. Paris: Vrin.

Norris, Pippa, und Ronald Inglehart. 2004. Sacred and secular. Religion and politics worldwide. Cambridge: Cambridge University Press.

Parsons, Talcott. 1935. The place of ultimate values in sociological theory. The International Journal of Ethics 45:282-316.

Parsons, Talcott. 1967. Christianity and modern industrial society. In Sociological theory and modern society, Talcott Parsons, 385-421. New York: The Free Press. 
Philipp, Thomas. 2009. Gesellschaft und Religion. Eine kritische Auseinandersetzung mit Habermas' Zeitdiagnose der postsäkularen Gesellschaft. Berliner Journal für Soziologie 19:55-78.

Philpott, Daniel. 2007. Explaining the political ambivalence of religion. American Political Science Review 101:505-525.

Philpott, Daniel. 2009. Has the study of global politics found religion? Annual Review of Political Science 12:183-202.

Pollack, Detlef. 1995. Was ist Religion? Probleme der Definition. Zeitschrift für Religionswissenschaft 3:163-190.

Pollack, Detlef. 2003. Säkularisierung - ein moderner Mythos? Studien zum religiösen Wandel in Deutschland. Tübingen: Mohr Siebeck.

Pollack, Detlef. 2009. Rückkehr des Religiösen? Tübingen: Mohr Siebeck.

Poulat, Emile. 1987. Liberté, Laicité. La guerre des deux France et le principe de la modernité. Paris: Cerf-Cujas.

Reckwitz, Andreas. 2001. Die Transformation der Kulturtheorie. Weilerswist: Velbrück Wissenschaft.

Robertson, Roland. 1987. From secularization to globalization. Journal of Oriental Studies 26:28-32.

Rosa, Hartmut. 1998. Identität und kulturelle Praxis. Politische Philosophie nach Charles Taylor. Frankfurt a. M.: Campus.

Schluchter, Wolfgang. 1979. Die Entwicklung des okzidentalen Rationalismus. Tübingen: Mohr.

Schluchter, Wolfgang. 1988. Religion und Lebensführung. Studien zu Max Webers Religions- und Herrschaftssoziologie. Frankfurt a. M.: Suhrkamp.

Schwinn, Thomas. 2001. Differenzierung ohne Gesellschaft. Umstellung eines soziologischen Konzepts. Weilerswist: Velbrück.

Schwinn, Thomas. 2009. Multiple modernities: Konkurrierende Thesen und offene Fragen. Ein Literaturbericht in konstruktiver Absicht. Zeitschrift für Soziologie 38:454-476.

Smith, Christian, (Hrsg.). 2003. The secular revolution: Power, interest, and conflict in the secularization of American public life. Berkeley: University of California Press.

Smith, Christian. 2010. What is a person? Rethinking humanity, social life, and the moral good from the person up. Chicago: University of Chicago Press.

Smith, Nicholas H. 2002. Charles Taylor: Meaning, morals and modernity. Cambridge: Polity Press.

Spohn, Willfried. 2008. Politik und Religion in einer sich globalisierenden Welt. Wiesbaden: VS Verlag für Sozialwissenschaften.

Stark, Rodney. 1999. Secularization, R.I.P. Sociology of Religion 60:249-274.

Taylor, Charles. 1975. Hegel. Cambridge: Cambridge University Press.

Taylor, Charles. 1992. Negative Freiheit. Zur Kritik des neuzeitlichen Individualismus. Frankfurt a. M.: Suhrkamp.

Taylor, Charles, (Hrsg.). 1993. Multikulturalismus und die Politik der Anerkennung. Frankfurt a. M.: Fischer. (1992).

Taylor, Charles. 1994. Quellen des Selbst. Die Entstehung der neuzeitlichen Identität. Frankfurt a. M.: Suhrkamp. (1989).

Taylor, Charles. 1995. Das Unbehagen in der Moderne. Frankfurt a. M.: Suhrkamp. (1991).

Taylor, Charles. 1996. Drei Formen des Säkularismus. In Das Europa der Religionen, Hrsg. Otto Kallscheuer, 217-246. Frankfurt a. M.: Fischer.

Taylor, Charles. 1997. Foreword. In The disenchantment of the world. A political history of religion, Marcel Gauchet, 9-15. Princeton: Princeton University Press.

Taylor, Charles. 1999. A catholic modernity? In A catholic modernity? Charles Taylor's marianist award lecture, Hrsg. James L. Heft, 13-38. Oxford: Oxford University Press.

Taylor, Charles. 2002. Die Formen des Religiösen in der Gegenwart. Frankfurt a. M.: Suhrkamp.

Taylor, Charles. 2004. Modern social imagineries. Durham: Duke University Press.

Taylor, Charles. 2007. The secular age. Cambridge: The Belknap Press of Harvard University. 
Tenbruck, Friedrich H. 1993. Die Religion im Maelstrom der Reflexion. In Religion und Kultur. Sonderheft der Kölner Zeitschrift für Soziologie und Sozialpsychologie, Hrsg. Jörg Bergmann, Alois Hahn und Thomas Luckmann, 31-67. Opladen: Westdeutscher Verlag.

Thomas, George M. 1996. Revivalism and cultural change. Christianity, nation building, and the market in the nineteenth century United States. Chicago: University of Chicago Press.

Torpey, John. 2010. A (post-)secular age? Religion and the two excetionalisms. Social Research 77:269-296.

Tschannen, Olivier. 1991. The secularization paradigm: A systematization. Journal for the Scientific Study of Religion 30:395-415.

Tschannen, Olivier. 1992. Les théories de la sécularisation. Geneve: Droz.

Tyrell, Hartmut. 2008. Kulturkämpfe in Frankreich und Deutschland und die Anfänge der Religionssoziologie. In Religionskontroversen in Frankreich und Deutschland, Hrsg. Matthias Koenig und Jean-Paul Willaime, 97-181. Hamburg: Hamburger Edition.

Van der Veer, Peter. 2001. Imperial encounters. Religion and modernity in India and Britain. Princeton: Princeton University Press.

Wallis, R., und Steve Bruce. 1992. Secularization: The Orthodox model. In Religion and Modernization, Hrsg. Steve Bruce, 8-30. Oxford: Oxford University Press.

Warner, R. Stephen. 1993. Work in progress towards a new paradigm for the sociological study of religion in the United States. American Journal of Sociology 98:1044-1093.

Warner, Michael, Jonathan Van Antwerpen, und Craig Calhoun (Hrsg.). 2010. Varieties of secularism in a secular age. Cambridge: Harvard University Press.

Wohlrab-Sahr, Monika, Uta Karstein, und Thomas Schmidt-Lux. 2009. Forcierte Säkularität: religiöser Wandel und Generationendynamik im Osten Deutschlands. Frankfurt a. M.: Campus.

Wuthnow, Robert. 1989. Communities of discourse. Ideology and social structure in the reformation, the enlightenment, and European socialism. Cambridge: Harvard University Press.

Zuckermann, Phil. 2009. Society without God. What the least religious nations can tell us about contentment. New York: New York University Press.

Matthias Koenig, 1971, Dr. phil., Professor für Soziologie, Institut für Soziologie, Georg-AugustUniversität Göttingen. Forschungsgebiete: Religionssoziologie, historisch-komparative Soziologie, soziologische Theorie. Veröffentlichungen u. a.: Menschenrechte. Campus, 2005; Religionskontroversen in Frankreich und Deutschland. Hamburger Edition, 2008 (hrsg. mit J.-P. Willaime); Institutional change in the world polity - international human rights and the construction of collective identities. International Sociology 23, 2008; International migration and the governance of religious diversity. McGill Queen's University Press, 2009 (hrsg. mit P. Bramadat); Religiosität türkischer Migranten im Generationenverlauf - ein Befund und einige Erklärungsversuche. Zeitschrift für Soziologie 38, 2009 (mit C. Diehl). 